\title{
Description of a new species of the leafhopper genus Zyginella Löw from Southwest China (Hemiptera, Cicadellidae, Typhlocybinae)
}

\author{
Yuehua Song ${ }^{1,2,3, \dagger}$, Zizhong $\mathrm{Li}^{1, \ddagger}$ \\ I Institute of Entomology, Guizhou University, Guiyang, Guizhou 550025, China 2 Institute of South China \\ Karst, Guizhou Normal University, Guiyang, Guizhou 550001, China 3 The State Key Laboratory Incubation \\ Base for Karst Mountain Ecology Environment of Guizhou Province, Guiyang, Guizhou 550001, China \\ † urn:lsid:zoobank.org:author:62E9B76A-24FF-4C61-9E33-E88393B5012A \\ † urn:lsid:zoobank.org:author:7B033BOF-8EF0-48D3-8F4D-DFA3A7F3FBD5 \\ Corresponding author: ZizhongLi (songyuehua@163.com; lizizhong38@163.com) \\ Academic editor: M. Webb | Received 30 September 2011 | Accepted 13 January 2012 | Published 31 January 2012 \\ urn:lsid:zoobank.org:pub:8298B213-E172-4FDA-9316-3A77887C15B3 \\ Citation: Song Y, Li Z (2012) Description of a new species of the leafhopper genus Zyginella Löw from Southwest China \\ (Hemiptera, Cicadellidae, Typhlocybinae). ZooKeys 168: 13-17. doi: 10.3897/zookeys.168.2171
}

\begin{abstract}
A new species, Zyginella menghaiensis sp. n. (Hemiptera: Cicadellidae: Typhlocybinae: Zyginellini), is described from China and a key to species of Zyginella from China is provided.
\end{abstract}

\section{Keywords}

Morphology, taxonomy

\section{Introduction}

The leafhopper genus Zyginella was established by Löw in 1885 . The genus belongs in the tribe Zyginellini of Typhlocybinae and consists of twenty-two species distributed in the Oriental, Palaearctic and Afrotropical Regions. Members of the genus can be distinguished by the distinct dark spot on the 3rd apical cell of the forewing (Fig. 3) and in the male genitalia by the male pygofer with short ventral caudal process and long macrosetae on the posterodorsal margin (Fig. 5) and style elongate, slender throughout length with truncate base (Fig. 7). 
Recent taxonomic work on the genus includes Dworakowska (1969, 1970, 1974, 1977), Chiang, Lee and Knight (1988) and Zhang (1990); up to now, eight species of Zyginella have been recorded from China in these studies. In the current work, a new species from Yunnan Province, China is described and illustrated and a key to Chinese species of Zyginella is given. All specimens examined are deposited to the collection of the Insititute of Entomology, Guizhou University, Guiyang, China (GUGC).

\section{Taxonomy}

\section{Zyginella Löw}

http://species-id.net/wiki/Zyginella

Zyginella Löw, 1885: 346; Dworakowska 1969: 433, 1970: 707, 1974: 161, 1977: 24;

Chiang et al. 1988: 109; Zhang 1990: 170. Type species: Zyginella pulchra Löw, 1885.

Pyramidotettix Matsumura, 1932: 59; Dworakowska 1970: 707; Chiang et al. 1988: 109; Yang 1965: 197. Type species: Conometopius citri Matsumura, 1907. Synonymized by Dworakowska 1970: 707.

Remmia Vilbaste, 1968: 91; Dworakowska 1970: 707; Chiang et al. 1988: 109. Type species: Remmia orbigera Vilbaste, 1968. Synonymized by Dworakowska 1970: 707.

Description. Forewing (Fig. 3) with distinct dark spot on 3rd apical cell.

Head (Fig. 1) acutely produced medially, about as wide as greatest width of pronotum; coronal suture prominent. Forewing (Fig. 3) with 1st apical cell short. Hind wing (Fig. 11) with submarginal vein confluent with $\mathrm{Cu}_{1}$ markedly distad of point of fusion of $\mathrm{Cu}_{1}$ with $\mathrm{M}_{3+4}$.

Male pygofer (Fig. 5) with short process on lateroventral margin and numerous long macrosetae on posterodorsal surface. Subgenital plate usually forming a pocketlike structure at tip or tapering towards apex (Fig. 6). Style (Fig. 7) broadened and truncate at base. Aedeagal shaft (Figs 8, 9) usually curved dorsally; preatrium long or short; dorsal apodeme narrow. Connective (Fig. 10) V-or Y-shaped; lateral arms long; central lobe absent.

Distribution. Oriental region, Palaearctic region, Afrotropical region.

\section{Key to Chinese species of the genus Zyginella (males only couplets 5-7)}

$1 \quad$ Vertex with black stripe between eyes subapically (Fig. 12) ........................2

- $\quad$ Vertex without black stripe between eyes subapically (Fig. 1) .......................4

2 Pronotum with two black transverse stripes ................. Z. citri (Matsumura)

- $\quad$ Pronotum without black transverse stripes ................................................. 3

3 Vertex and pronotum with pair of dark spots respectively ..... Z . mali (Yang)

- $\quad$ Vertex and pronotum without spots..................................... minuta (Yang) 
$4 \quad$ Forewings without a large rhombus-like patch along inner margin subbasally

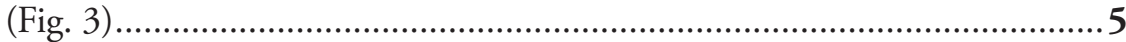

- $\quad$ Forewings with a large rhombus-like patch along inner margin subbasally

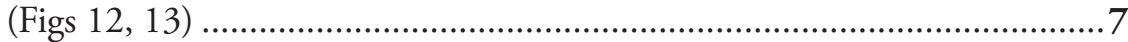

5 Aedeagus preatrium well developed, about as long as length of aedeagal shaft

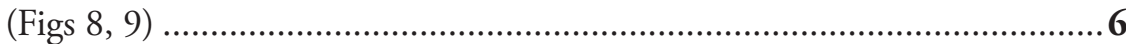

- $\quad$ Aedeagus preatrium vestigial ..................................... Z. orla Dworakowska

6 Aedeagal shaft with a single short dorsal process subapically (Figs 8, 9)

Z. menghaiensis sp. $\mathbf{n}$.

- $\quad$ Aedeagal shaft without a single short dorsal processes subapically

Z. tsauri Chiang, Hsu \& Knight

$7 \quad$ Pygofer lobe with a hook-like process caudo-dorsally..... Z. punctata Zhang

Pygofer lobe without hook-like process caudo-dorsally

Z. taiwana Chiang, Lee \& Knight

\section{Zyginella menghaiensis Song \& $\mathrm{Li}$, sp. $\mathrm{n}$.}

urn:lsid:zoobank.org:act:090D3B2C-B357-4E11-9F81-AC71C63C6743

http://species-id.net/wiki/Zyginella_menghaiensis

Figures $1-11$

Description. Head and thorax yellowish brown; vertex with lateral margins with soft red tinge; eyes brownish grey; pronotum brownish with two longitudinal darker stripes; scutellum with basal triangles testaceous. Forewing (Fig. 3) reddish brown near base, dark red between 4th apical cell and brochosome-field and light brown around apex; 3rd apical cell with a blackish brown spot.

Coronal suture (Fig. 1) extending nearly to anterior margin of vertex. Forewing (Fig. 3) with 3rd apical cell not petiolate at base.

Abdominal apodemes (Fig. 4) slender, slightly extended beyond 4th sternite.

Pygofer lobe (Fig. 5) broad, with a large sclerotized process near dorsal margin and another process arising from about ventro-caudal margin; six long macrosetae distributed along caudal margin and numerous short microsetae scattered on lateral surface. Subgenital plate (Fig. 6) long, gradually tapered towards apex and curved apically, beak-like; with three long macrosetae along upper margin. Style (Fig. 7) elongate, slender throughout length with truncate base. Aedeagal shaft (Figs 8, 9) curved dorsad in lateral view with single small dorsal process subapically; gonopore large, apical on ventral surface with small tooth on each lateral margin; preatrium long, about as long as aedeagal shaft; dorsal apodeme narrow. Connective (Fig. 10) Y-shaped with very short stem and long strongly divergent lateral arms; central lobe absent.

Measurement. Body length males 2.9 3.1 mm.

Type material. Holotype, male, China: Yunnan Province, Menghai County, 23 July 2008, coll. YUE-HUA SONG. Paratypes: two males, same date as holotype. 


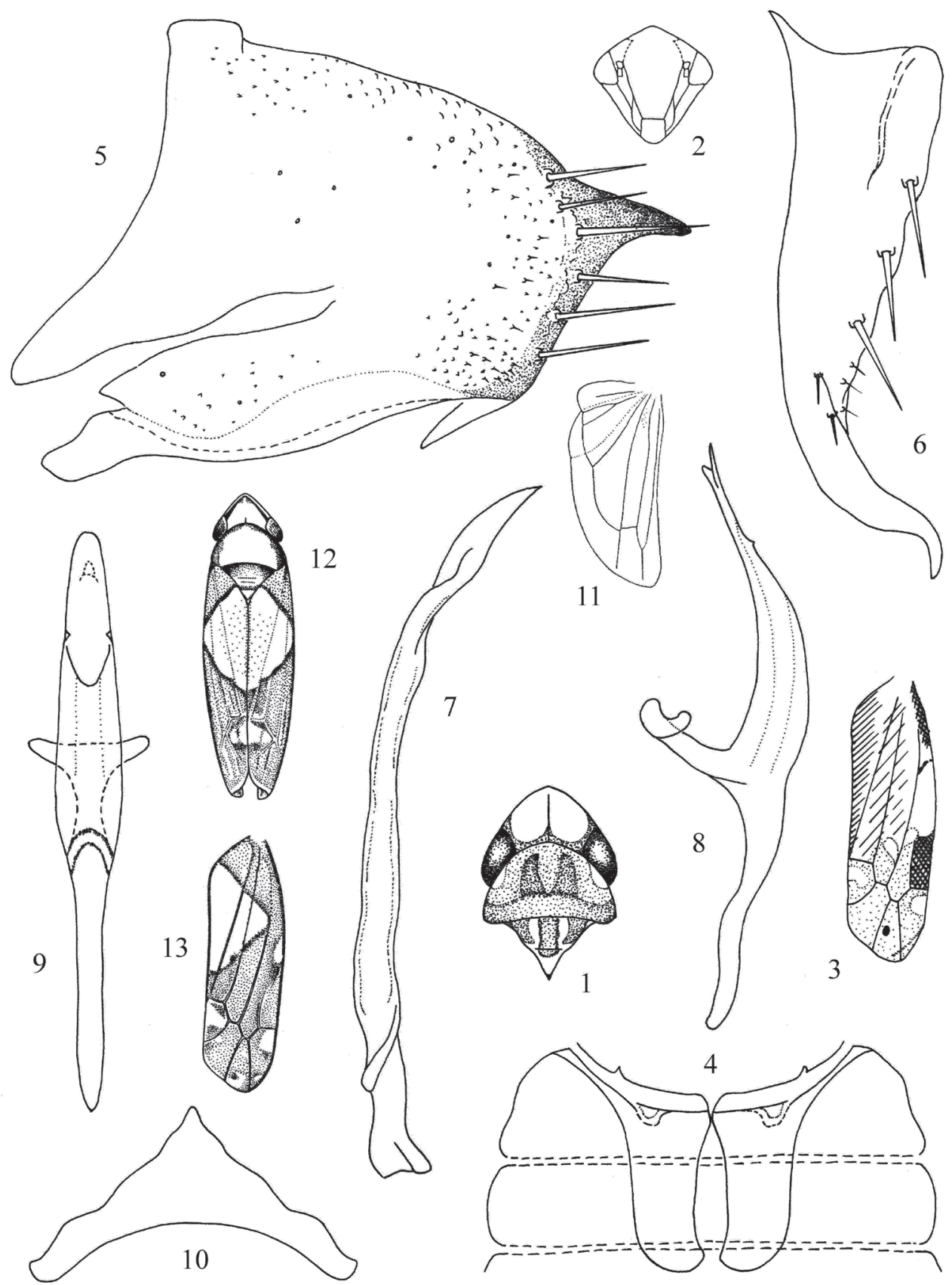

Figures I-13. Zyginella species I-I I Zyginella menghaiensis sp. n. I Head and thorax, dorsal view 2 Face 3 Forewing 4 Abdominal apodemes 5 Pygofer lobe, lateral view 6 Subgenital plate 7 Style 8 Aedeagus, lateral view 9 Aedeagus, ventral view 10 Connective I I Hindwing I 2-I3 Zyginella minuta (after Yang, 1965) 12 Adult, dorsal view 13 Forewing. 
Remarks. The new species is similar to Z. tsauri Chiang, Hsu and Knight (1989), but the forewing has a large dark costal patch (Fig. 3) and the aedeagus has a single short dorsal process subapically and a small tooth on each lateral margin of the gonopore (Figs 8, 9).

Etymology. The new species is named for its type locality: Menghai.

\section{Acknowledgements}

We would like to express our sincere gratitude to Mr. M. D. Webb (Natural History Museum, London, UK) for his detailed remarks and suggestions on the manuscript. The project was supported partly by the "Scientific Research Foundation for Doctor's Degree Members, Guizhou Normal University: Taxonomic Study of Erythroneurini and Zyginellini from South China Karst Area", the "Social Development and Science \& Technology of People's Livelihood Program of Guiyang City (No. 201110362)", the "Key Technologies R\&D Program of Guizhou Province (SY[2010]3068)" and the "Guizhou Provincial Natural Science Foundation of China (No. [2010]2063)".

\section{References}

Chiang CC, Hsu TC, Knight WJ (1989) Studies on Taiwanese Typhlocybinae (Homoptera: Cicadellidae). (II) Tribe Typhlocybini and two new species of Zyginellini. Journal of Taiwan Museum 42(1): 143-144.

Chiang CC, Lee PP, Knight WJ (1988) Studies on Taiwanese Typhlocybinae (Homoptera: Cicadellidae). (I) Tribe Zyginellini. Journal of Taiwan Museum 41(1): 103-112.

Chou I, Zhang YL (1985) On the tribe Zyginellini from China (Homoptera: Cicadellidae: Typhlocybinae). Entomotaxonomia 7(4): 292. Yang CK (1965) Two new leafhoppers of the genus Pyramidotettix (Homoptera: Jassidae) injuring apple trees. Acta Entomologica Sinica 14(2): 196-202.

Dworakowska I (1969) On the genera Zyginella Löw and Limassolla Dlab. (Cicadellidae, Typhlocybinae). Bulletin de l'Academie Polonaise des Sciences, Serie des Sciences Biologiques 17(7): 433-435.

Dworakowska I (1970) On some genera of Typhlocybini and Empoascini (Auchenorrhyncha, Cicadellidae, Typhlocybinae). Bulletin de l'Academie Polonaise des Sciences, Serie des Sciences Biologiques 18(11): 707-710.

Dworakowska I (1974) Contribution à la faune du Congo (Brazzaville). Mission A. Villiers et A. Descarpentries. CIX. Hémiptères Typhlocybinae. Bull. IFAN. (A). 36(1): 161-164.

Dworakowska I (1977) On some Typhlocybinae from Vietnam (Homoptera: Cicadellidae). Folia Entomologica Hungarica 30(2): 24-28.

Zhang YL (1990) A taxonomic study of Chinese Ciadellidae (Homoptera). Tianze Eldonejo. Yangling, Shaanxi, China 170-173. 